Research Article

\title{
Effect of Airflow Field in the Tangential-Longitudinal Flow Threshing and Cleaning System on Harvesting Performance
}

\author{
Taibai Xu $(\mathbb{D})$ and Yaoming $L i$ (iD \\ School of Agricultural Equipment Engineering, Jiangsu University, Zhenjiang 212013, Jiangsu, China \\ Correspondence should be addressed to Taibai Xu; xutaibai84664379@163.com and Yaoming Li; ymli@ujs.edu.cn
}

Received 15 February 2020; Revised 28 June 2020; Accepted 10 July 2020; Published 5 August 2020

Academic Editor: Pietro Russo

Copyright (c) 2020 Taibai Xu and Yaoming Li. This is an open access article distributed under the Creative Commons Attribution License, which permits unrestricted use, distribution, and reproduction in any medium, provided the original work is properly cited.

\begin{abstract}
The threshing and cleaning device in the grain combine harvester is located in the same airtight space, and the air flow field in it should also be studied and tested as a whole system. In order to study the distribution of air flow field and the influence of working parameters on the air flow field in the internal space of threshing and cleaning system, the method of predicting harvest performance indexes (grain loss rate and grain impurity rate) by air flow field analysis was explored. First of all, taking the longitudinal grain combine harvester of our research group as the test object and taking the rotating speed of centrifugal fan, the angle of fan plate, the opening of chaffer, and the rotating speed of threshing cylinder as the research factors, the internal space flow channel model of threshing and cleaning system under different working conditions was established and CFD software was used to simulate and analyze the air flow field. At the same time, the hot wire anemometer is used to measure and verify the distribution of air flow field in the threshing and cleaning system under various working conditions. Then, the harvest performance index of the threshing and cleaning system under the rated feeding rate is tested under the corresponding working conditions to find the relationship between the distribution of air flow field and harvest performance, put forward the corresponding analysis and prediction methods, and establish the mathematical relationship model between the simulated air flow field and harvest performance index. The results of simulation and experiment show that the average air velocity can more accurately reflect the cleaning performance. The mathematical function of the relation curve is $Y=11.71 X-4.76$, and the prediction error is within $9.4 \%$. The air velocity in the middle area of the vibrating screen is approximately in proportion to the cleaning performance, which provides the theoretical and experimental basis for the design of the threshing and cleaning device and the adjustment of the working parameters in the field harvest. In addition, it can save the design time and cost and reduce the seasonal impact of field experiment.
\end{abstract}

\section{Introduction}

Threshing and cleaning device is the core part of the rice combine harvester and the "digestive system" of the whole machine, in which the threshing device takes off the grains from the rice ears, forming the mixture of grain and material other than grain mixture Delete "(grain MOG mixture for short)" which contains grains, short stalks, light which contains grains, short stalks, light impurities, and a small amount of long stalks. In the cleaning device, the grains in grain and material other than grain mixture are separated from the short stalks and light impurities, and the short stalks and light impurities are blown out of the machine to get clean grains [1]. At present, the high-efficiency threshing and cleaning device at home and abroad often adopts the structure form of cutting longitudinal flow threshing roller plus multiair channel cleaning device [2], which has high efficiency of threshing and cleaning and strong adaptability to the harvest operation with large feed volume, and has been widely recognized by the market. The threshing and cleaning device in this structure is located in the same closed space, and the air flow field distribution in the internal space may directly affect the key performance of the combine harvester, such as the grain loss rate and the grain impurity content, while the air flow field distribution is closely related to the main working parameters of the combine harvester 
(threshing drum speed, centrifugal fan speed, air distributor angle, fish scale screen opening, etc.) [3], so it is meaningful to study the airflow field inside the threshing and cleaning device as a whole system.

Many scholars, John Deere, Case, Claas, and Kubota, and other harvest machinery companies have conducted much theoretical and experimental research on the air flow field of threshing and cleaning device to improve the harvest performance and efficiency. An effective cleaning system with more than one fan (HC1100, Iseki Co., Tokyo, Japan) or a fan with multiple outlets has been used in modern grain compounds to improve cleaning efficiency, such as the John Deere S670, Massey Ferguson 9500 series, and Class Tucano 470, Design new cleaning device structure to improve cleaning efficiency [4]. If the sieve overloads first, the tailings returns will overload and plug such that the cleaning performance declines significantly [5-7]. Therefore, an improved cleaning system needs to be robust against uneven distribution of the material on the sieve surface [8]. New fans are used to increase the uniformity of air flow distribution and reduce the air flow attenuation at the tail of vibrating screen $[9,10]$. This paper describes the turbulent vortex of the air flow in the cleaning device of the combine harvester and analyzes its formation reason and influence on the cleaning performance $[11,12]$. CFD method is used to analyze the distribution of air flow field in the cleaning device, providing reference for the design of cleaning device structure [13-15]. Optimize the combination of cleaning working parameters and carry out field cleaning orthogonal test $[16,17]$.

However, the existing research on the simulation and test of the airflow field in the combine harvester is limited to the cleaning device, not considering the flow of gas in the threshing device, nor considering the interaction between the airflow generated by the threshing device and the cleaning device. In addition, in the process of optimizing the parameters of the threshing and cleaning device, it often depends on experience or test, lacking the corresponding theoretical basis, and the test time is long, which is restricted by the grain growth cycle. Therefore, in this paper, the overall air flow field distribution in the internal space of the threshing and cleaning device with longitudinal flow is studied. Through CFD numerical simulation and grain harvest experiment, the influence rule of the change of air flow field on the harvest performance index (grain loss rate and grain impurity rate) is explored, and the mathematical relationship model between the air flow field and the harvest performance index in the threshing and cleaning system is established. When optimizing the parameters or improving the structure of the threshing and cleaning device, this research method can be used to predict the harvest performance, shorten the design and development time of the combine harvester, and reduce the seasonal impact of the field test.

\section{Materials and Methods}

2.1. Experimental Design. The research object is the highefficiency tangent longitudinal flow threading and cleaning system developed by our research group, as shown in Figure 1. This system has high efficiency and strong adaptability to the harvest conditions of large feeding amount and wet crops, which is suitable for the harvest operations of paddy fields in South China [18, 19]. According to the adjustment range of its actual working parameters, the air flow field simulation's four-factor and four-level test scheme is developed through JMP software, as shown in Table 1. The test scheme can ensure the accuracy of the test analysis results on the premise of reducing the number of tests. Each group of tests was repeated three times, and the results were averaged.

\subsection{Airflow Field Simulation Process}

2.2.1. Airflow Field Simulation Theory. During the functioning of the threshing and cleaning system, airflow is usually in a turbulent state. The standard turbulence model $k-\varepsilon$ proposed by Jones and Launder is the most widely used of these models [20-22]. Specifically, the differential equations are introduced for the turbulent kinetic energy, $k$, and the turbulent kinetic energy dissipation ratio, $\varepsilon$. Both the turbulent velocity scale transport and the turbulent length scale transport are taken into account in the model. It is suitable for certain complex three-dimensional turbulent flows, by describing the real physical processes of various flows. Additionally, the basic form of the model is relatively simple, and it shows a high calculation accuracy. The standard $k-\varepsilon$ turbulence model has been successfully applied to agricultural machinery in the calculation analysis of two-phase gas-solid flows [23, 24]. In the present study, this model is again used for the calculation of the airflow field in the threshing and cleaning system.

The control equation is

$$
\begin{aligned}
\frac{\partial}{\partial t}(\rho k)+\frac{\partial}{\partial x_{i}}\left(\rho k u_{i}\right)= & \frac{\partial}{\partial x_{j}}\left[\left(\mu+\frac{\mu_{t}}{\sigma_{k}}\right) \frac{\partial k}{\partial x_{j}}\right]+G_{k}+G_{b} \\
& -\rho \varepsilon-Y_{M}+S_{k}, \\
\frac{\partial}{\partial t}(\rho \varepsilon)+\frac{\partial}{\partial x_{i}}\left(\rho \varepsilon u_{i}\right)= & \frac{\partial}{\partial x_{j}}\left[\left(\mu+\frac{\mu_{t}}{\sigma_{k}}\right) \frac{\partial \varepsilon}{\partial x_{j}}\right] \\
& +C_{1 \varepsilon} \frac{\varepsilon}{k}\left(G_{k}+C_{3 \varepsilon} G_{b}\right)-C_{2 \varepsilon} \rho \frac{\varepsilon^{2}}{k}+S_{\varepsilon},
\end{aligned}
$$

where

$$
\begin{aligned}
\mu_{t} & =\rho C_{\mu} \frac{k^{2}}{\varepsilon} \\
G_{k} & =\mu_{t}\left(\frac{\partial u_{i}}{\partial x_{j}}+\frac{\partial u_{j}}{\partial x_{i}}\right) \frac{\partial u_{i}}{\partial x_{j}}, \\
G_{b} & =\beta g_{i} \frac{\mu_{t}}{\operatorname{Pr}_{t}} \frac{\partial T}{\partial x_{i}}, \\
Y_{M} & =2 \rho \varepsilon M_{t}^{2},
\end{aligned}
$$




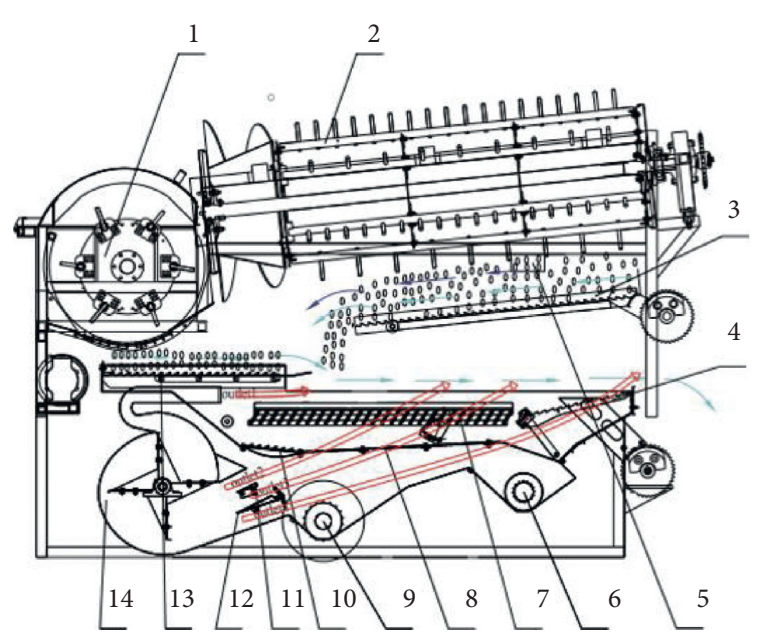

FIgURE 1: Schematic showing new type of rice threshing and cleaning system: $1=$ tangential thresher, $2=$ longitudinal threshing cylinder, $3=$ return pan, $4=$ tailings sieve, $5=$ tailings window, $6=$ tailings auger, $7=$ chaffer, $8=$ lower sieve, $9=$ clean grain auger, $10=$ lower pan, $11=$ upper airflow deflector, $12=$ lower airflow deflector, 13 = oscillating pan, and $14=$ multiduct centrifugal fan.

TABLe 1: Four-factor and four-level test plan.

\begin{tabular}{lcccc}
\hline & $\begin{array}{c}\text { Fan } \\
\text { speed } \\
\left(\mathrm{m} \cdot \mathrm{s}^{-1}\right)\end{array}$ & $\begin{array}{c}\text { Angle of air } \\
\text { distributor }\left(^{\circ}\right)\end{array}$ & $\begin{array}{c}\text { Chaffer } \\
\text { opening } \\
(\mathrm{mm})\end{array}$ & $\begin{array}{c}\text { Speed of tangential } \\
(\text { longitudinal flow } \\
\text { drum }\left(\mathrm{m} \cdot \mathrm{s}^{-1}\right)\end{array}$ \\
\hline 1 & 1500 & 24 & 26 & $620(580)$ \\
2 & 1200 & 28 & 26 & $720(674)$ \\
3 & 1500 & 28 & 22 & $770(720)$ \\
4 & 1500 & 22 & 20 & $720(674)$ \\
5 & 1200 & 24 & 22 & $670(627)$ \\
6 & 1200 & 26 & 20 & $620(580)$ \\
7 & 1400 & 24 & 20 & $770(720)$ \\
8 & 1200 & 22 & 24 & $770(720)$ \\
9 & 1500 & 26 & 24 & $670(627)$ \\
10 & 1300 & 24 & 24 & $720(674)$ \\
11 & 1400 & 26 & 22 & $720(674)$ \\
12 & 1400 & 28 & 24 & $620(580)$ \\
13 & 1400 & 22 & 26 & $670(627)$ \\
14 & 1300 & 26 & 26 & $770(720)$ \\
15 & 1300 & 28 & 20 & $670(627)$ \\
16 & 1300 & 22 & 22 & $620(580)$ \\
\hline
\end{tabular}

where $\mu_{t}$ is the viscosity of turbulence in units of Pa.s; $\rho$ is air density in units of $\mathrm{kg} \cdot \mathrm{m}^{-3} ; G_{k}$ is the generation item of turbulent kinetic energy, $k$, caused by the mean velocity gradient; $G_{b}$ is the generation item of turbulent kinetic energy, $k$, caused by buoyancy; $Y_{M}$ is the contribution of fluctuation distribution in compressible turbulence; $S_{i}, S_{k}$, are $S_{\varepsilon}$ are source items; Prt is the turbulent Prandtl number; $\beta$ is the coefficient of explatesion due to heat; and $M_{t}$ is the Mach number. In the fluent model of constant value, $C_{1 \varepsilon}=1.44, C_{2 \varepsilon}=1.92, C_{\mu}=0.09, \sigma_{k}=1.0, \sigma_{\varepsilon}=1.3$, Prt $=0.85$.

2.2.2. Establishment of the Runner Model. The SolidWorks software was used to establish the internal space flow channel models of 13 threshing and cleaning devices under different working conditions. There is no need to set up additional runner model for the two factors of centrifugal fan speed and threshing drum speed, because the speed can be set directly in the simulation software, only considering the different combinations of the angle of the fan plate and the opening of the chaffer in the test scheme. The model has a total width of $990 \mathrm{~mm}$, a total length of $2292 \mathrm{~mm}$, a diameter of $500 \mathrm{~mm}$, a diameter of $605 \mathrm{~mm}$, and an angle of $6^{\circ}$ between the axis and the horizontal plane. Figure 2 is the schematic diagram of flow channel model of threshing and cleaning device under working condition 5.

2.2.3. Grid Generation. The ICEM module of Ansys Workbench software is used to divide the grid. Considering the large volume and internal complexity of the runner model, the unstructured tetrahedral grid is divided for the runner model. Because the runner model has the rotating motion of centrifugal fan impeller, cutting flow threshing drum and longitudinal axial flow threshing drum, the multiarea grid division method is adopted to prepare for the MRF multicoordinate system model calculation. The runner model is divided into four areas, namely, fan impeller area, cutting flow threshing drum area, longitudinal axial flow threshing drum area, and cleaning chamber area. At the same time, four air inlets and one air outlet are set (Figure 3). The mesh interface between adjacent areas is set as interface. The mesh of the above four areas is divided, and the centrifugal fan blades, threshing roller teeth, concave screen, scale screen, woven screen, and tail screen are divided into face mesh and locally densified to improve the quality and accuracy of the mesh. Finally, the mesh of the four areas is combined. After merging the grids, the total number of grids of the seven channel models is between 22,728,519 and $22,934,564$, respectively.

2.2.4. Simulation Parameter Setting. Without considering the influence of temperature, humidity, and other environmental factors and ignoring the viscous force between gas molecules, the gas in the tangential flow threshing and cleaning device is approximately incompressible, and the standard turbulence calculation model is adopted. The grid area of impeller, tangential threshing drum, screw feed head, and longitudinal axial threshing drum of multichannel centrifugal fan is set as MRF rotation area, and the corresponding rotation coordinate axis and rotation speed are set; the boundary conditions of three air inlets of multichannel centrifugal fan are set as pressure inlet $0 \mathrm{~Pa}$, the boundary conditions of air inlet of threshing device are set as pressure inlet $0 \mathrm{~Pa}$, and the air outlet of model tail is set as pressure inlet $0 \mathrm{~Pa}$. The boundary condition of the port is set as $0 \mathrm{~Pa}$ at the pressure outlet [16]. In the solution control, the relaxation factor is set by fluent default, the residual is set to 0.0001 , and the number of iteration steps is set to 3000 .

2.3. Flow Field Verification Test. In order to verify the accuracy of air flow field simulation, the air flow velocity measurement test in the threshing and cleaning device with 


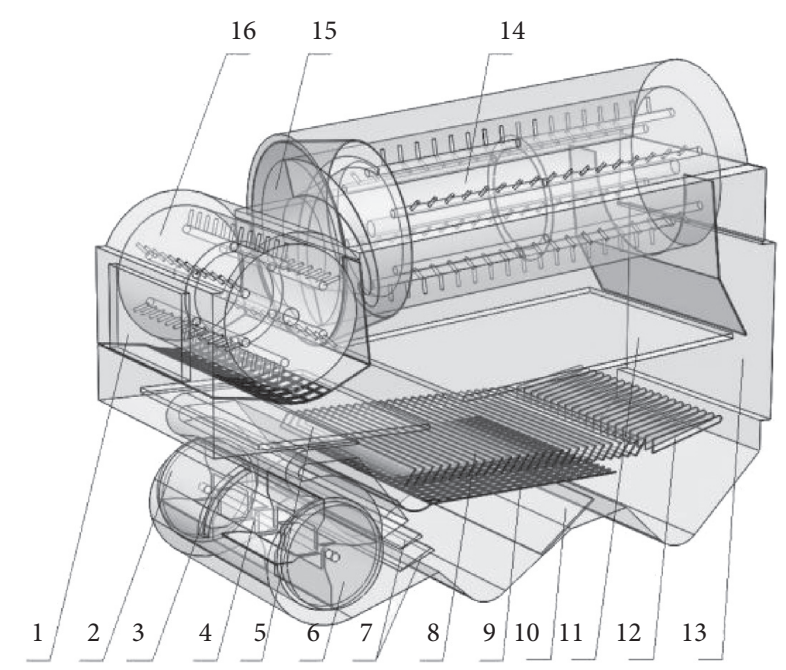

FIgURE 2: Threshing and cleaning device flow-path model: $1=$ upper air inlet, $2=$ left air inlet, $3=$ middle air inlet, $4=$ impeller area, $5=$ oscillating pan, $6=$ right air inlet, $7=$ airflow deflector, $8=$ vibrating sieve (louver sieve), $9=$ vibrating sieve (woven sieve), $10=$ rubber sheet, $11=$ return pan, $12=$ tailing sieve, $13=$ air outlet, $14=$ longitudinal threshing cylinder area, $15=$ screw feeding head area, and $16=$ tangential threshing cylinder area.

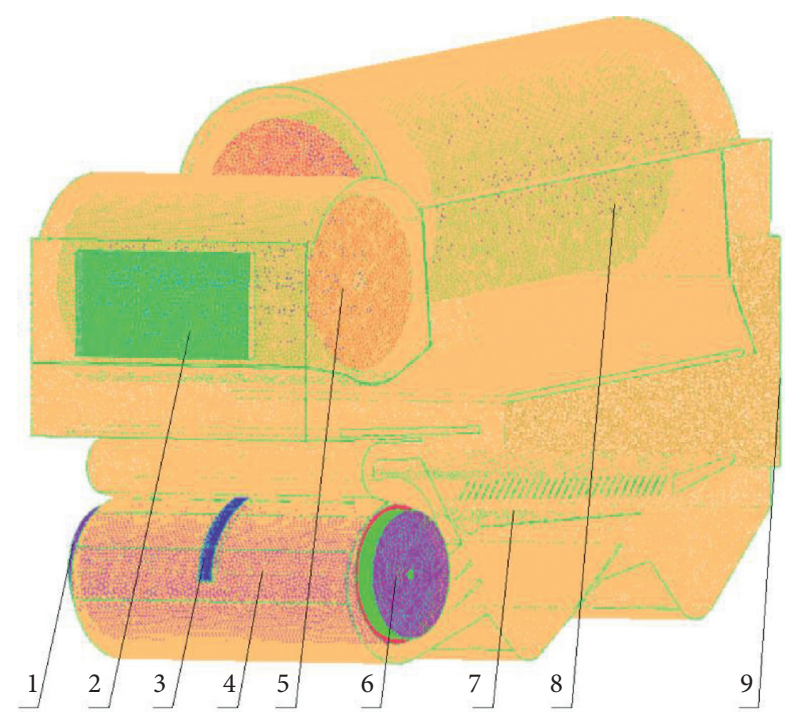

Figure 3: Mesh model: $1=$ left air inlet, $2=$ upper air inlet, $3=$ middle air inlet, $4=$ impeller area, $5=$ tangential threshing cylinder area, $6=$ right air inlet, $7=$ cleaning-room area, $8=$ longitudinal threshing cylinder area, and $9=$ air outlet.

longitudinal flow was carried out. In consideration of the actual internal structure and measurement space of the vertical flow threshing and cleaning device, two horizontal measurement surfaces are arranged above and below the vibrating screen, and one horizontal measurement surface is arranged below the cutting flow threshing drum, totaling three horizontal measurement surfaces. The first measuring surface is $80 \mathrm{~mm}$ above the upper screen (scale screen) of the vibrating screen, the second measuring surface is $15 \mathrm{~mm}-70 \mathrm{~mm}$ below the lower screen (woven screen) of the vibrating screen, the third measuring surface is $20 \mathrm{~mm}-130 \mathrm{~mm}$ below the cutting threshing drum, and the size and distribution of the three measuring planes are the same. Take the leftmost front of the shale shaker as the coordinate origin, extend horizontally along the left side of the screen face to the right side of the screen face in the positive $Y$-axis direction, extend longitudinally along the screen face to the tail in the positive $X$-axis direction, and take the vertical direction of the longitudinal flow threshing and cleaning device as the $Z$-direction. 25 measuring points are arranged uniformly in $X$ - and $Y$-directions in 3 measuring planes. The distribution diagram of measuring surface and measuring points is shown in Figures 4 and 5 .

At present, the main methods of measuring air velocity in scientific research include hot wire anemometer, PIV technology, and laser Doppler testing technology [25]. Because of the large structure size, low cost, small volume, small interference of convection field, and high precision of hot wire anemometer, the hot wire anemometer (Nanjing Nengzhao Technology Co., Ltd., VS110 type) is used to measure the air velocity of internal measuring points in this test. The hot wire anemometer is installed on the retractable rotating bench, and it is inserted into the measuring hole on the side of the threshing and cleaning device of the longitudinal flow according to the distribution of the measuring points, so as to realize the point distribution measurement. The measuring data can be read directly through the screen, as shown in Figure 6. In order to ensure the accuracy of measurement results and eliminate accidental errors, a group of measurement tests are repeated three times, and the average value of three tests is taken as the measurement results.

\subsection{Cleaning Performance Test}

2.4.1. Test Material Characteristics. The experimental rice variety is "Zhendao 18," the moisture content of grain is $24.9 \%$, and the moisture content of stem is $50.4 \%$. Rice exfoliated mixture includes full grain, thin grain, short stem (length less than or equal to $50 \mathrm{~mm}$ ), light miscellaneous surplus, and a small amount of long stem (length greater than $50 \mathrm{~mm}$ ). The suspension speed of the main components was measured by dfpf- 25 material floating characteristic measuring device (Figure 7). 20 samples were taken for each mixture component. After multiple measurements of each material, the maximum and minimum values of the floating speed were obtained, as shown in Table 2.

It can be seen from Table 2 that in theory, when the air velocity above the vibrating screen meets the condition of $5.8 \mathrm{~m} / \mathrm{s}<V<8.8 \mathrm{~m} / \mathrm{s}$, the materials in the cleaning room can be effectively stratified, diffused, and screened, while, in practice, the movement of the vibrating screen and the shaking plate plays a certain role in promoting the stratification and diffusion of the mixture, so the air velocity at the cleaning screen surface should be $3.0 \mathrm{~m} / \mathrm{s} \sim 6.0 \mathrm{~m} / \mathrm{s}$ among them, there are many long stalks and debris at the tail of screen face, and the air flow speed should be between $5.0 \mathrm{~m} / \mathrm{s}$ 


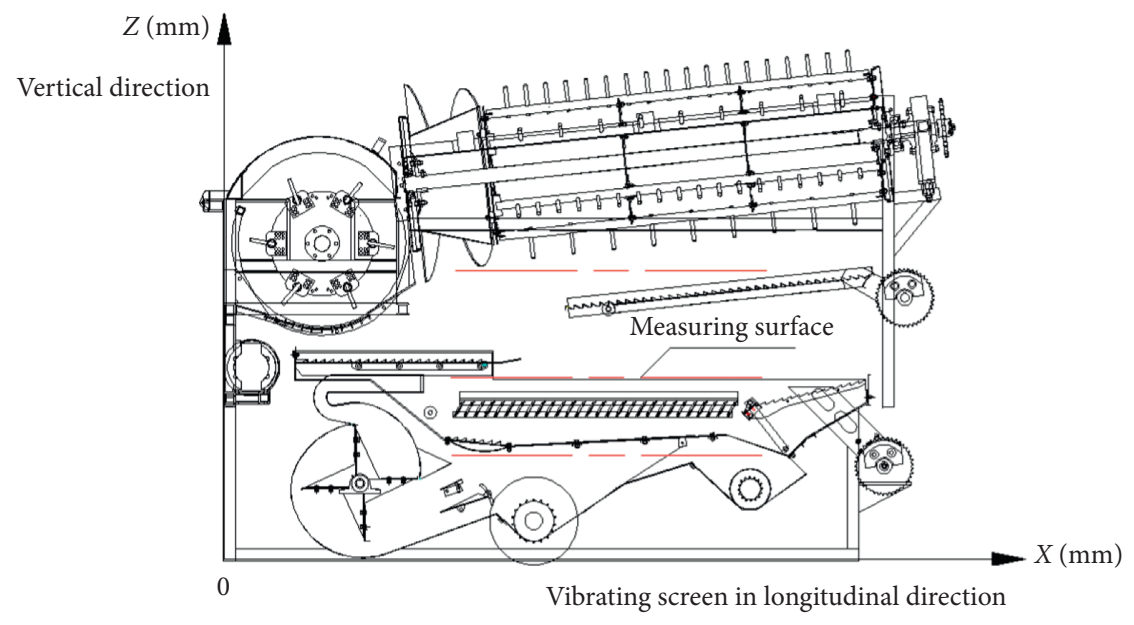

FIGURE 4: Distribution of measuring surface.

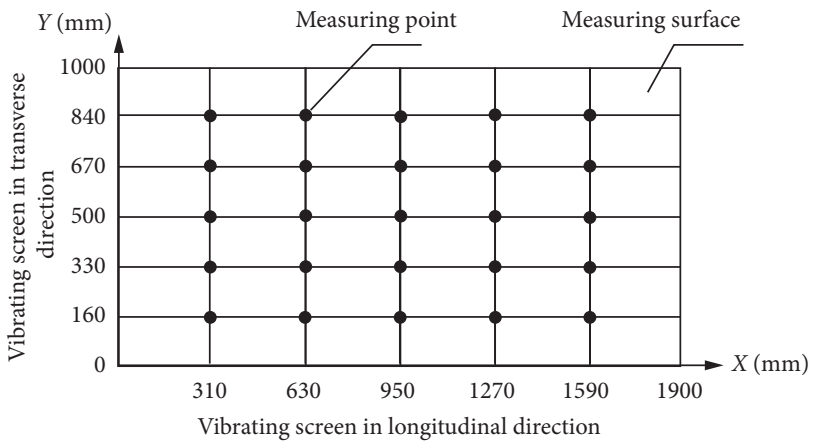

Figure 5: Distribution diagram of measuring points.

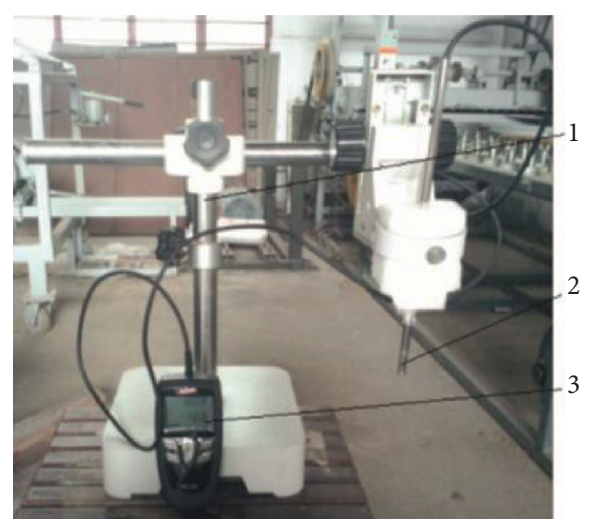

Figure 6: Airflow velocity measuring device: 1, adjustable holder; 2, hotwire anemometer; and 3, display unit.

and $6.0 \mathrm{~m} / \mathrm{s}$, so that the long stalks and debris can be effectively blown out of the machine [17].

2.4.2. Cleaning Test Device. The 4lz-3.5 slitting and longitudinal flow combine harvester produced by Wuxi United Harvester Co., Ltd. was used as the experimental prototype for field harvest test. The loss collection device was installed behind the vibrating screen of TH988 slitting and longitudinal flow full feeding combine harvester, as shown in
Figure 8. The mixture blown out from the oilcloth is collected, and the grains in the blown out mixture are cleaned out by a small cleaning fan, and then the grains are weighed to obtain the grain quality. Then, the total mass of grains in the grain tank and the total mass of impurities in the grain tank and stalks are weighed to calculate the cleaning performance evaluation indexes such as cleaning loss rate and grain impurity rate. Repeat three times for each group of tests, and take the average value of the final test results.

\section{Test Results and Analysis}

3.1. Simulation Test Results of the Air Flow Field. After the fluent calculation converges, the CFX postprocessing software is used to process and analyze the air flow field, and the distribution of the overall air flow field inside the threshing and cleaning device of the tangential and longitudinal flow can be obtained. Figures 9 and 10 are the streamline diagram and velocity cloud diagram under the rated working condition.

According to the simulation results, the following can be concluded:

(1) The rotation of the tangential flow and longitudinal axial flow threshing roller will produce certain air flow; especially the wind speed at the screw feed head is relatively large, about $7.0 \mathrm{~m} / \mathrm{s} \sim 8.5 \mathrm{~m} / \mathrm{s}$, which increases the auxiliary feeding effect of the material transfer from the circular motion in the tangential flow threshing device to the spiral motion in the longitudinal axial flow threshing device, which is conducive to reducing the blockage at the transition point. In addition, blocked by the separation concave, the air flow of the tangential flow and longitudinal axial flow threshing device is mainly concentrated in the interior of the threshing space, only a small part of the air flow flows to the cleaning device, and there is a small amount of intersection with the cleaning air flow.

(2) The air flow $(3.5 \mathrm{~m} / \mathrm{s} \sim 6.8 \mathrm{~m} / \mathrm{s})$ produced by the air outlet of the multichannel centrifugal fan is helpful 


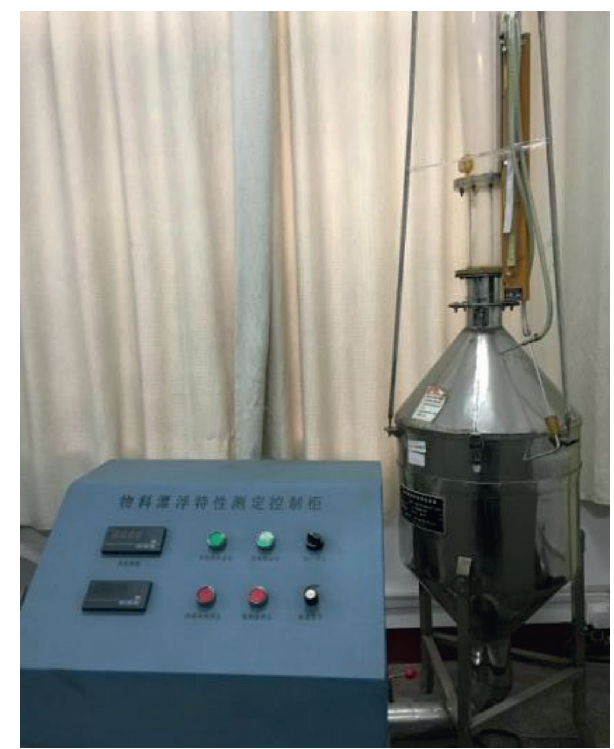

FIgURE 7: Material floating velocity measurement device.

TABLE 2: Floating velocity of grain mixture.

\begin{tabular}{lcc}
\hline Material & $\begin{array}{c}\text { Maximum floating } \\
\text { velocity }\left(\mathrm{m} \cdot \mathrm{s}^{-1}\right)\end{array}$ & $\begin{array}{c}\text { Minimum floating } \\
\text { velocity }\left(\mathrm{m} \cdot \mathrm{s}^{-1}\right)\end{array}$ \\
\hline Full grain & 10.1 & 8.8 \\
Thin grain & 4.2 & 1.5 \\
Long stem & 5.8 & 3.8 \\
Short stem & 4.0 & 2.3 \\
Light impurity & 2.8 & 0.9 \\
\hline
\end{tabular}

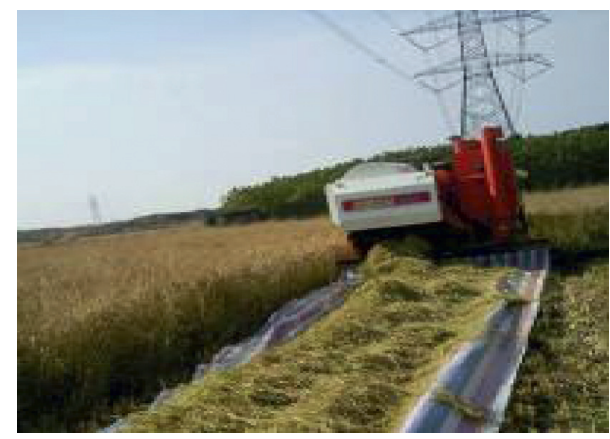

Figure 8: Cleaning performance test.

to blow the light debris (floating speed $\leq 2.8 \mathrm{~m} / \mathrm{s}$ ) at the front above the vibrating screen to the outside of the fan, and it is helpful to blow the short straw and the Shrunken Valley (floating speed $\leq 4.2 \mathrm{~m} / \mathrm{s}$ ) to the screen tail and has little influence on the movement of the grain (floating speed $\geq 8.8 \mathrm{~m} / \mathrm{s}$ ). The air flow generated by the three air outlets below covers the whole shale shaker, making the air flow velocity at the middle and rear of the screen surface $4.2 \mathrm{~m} /$ $\mathrm{s}-7.5 \mathrm{~m} / \mathrm{s}$, which is conducive to the layered diffusion of materials on the screen; the air flow velocity at the tail of the shale shaker rises to $5.2 \mathrm{~m} / \mathrm{s}-6.0 \mathrm{~m} / \mathrm{s}$, which is conducive to the discharge of short straw, shrunken grain, and long straw out of the machine. 8

3.2. Airflow Field Measurement Verification Test Results. Since the measurement results of three repeated tests in each group of measurement tests are very close, and the error is between $0.2 \%$ and $1.5 \%$, the average value of the repeated test results is taken. According to the measuring points arranged in the air flow field measurement test, the air flow velocity at the same position is extracted from the simulation results. The comparison between the simulation results of the gas flow field and the measurement test results is made by using Origin software. Because there are many measuring points, the average value of the air flow velocity of the measuring points in the transverse direction of the threshing and cleaning device is taken as the comparison result. Figure 11 shows the comparison of measurement test and simulation results under condition 5 .

The following can be concluded.

(1) In the longitudinal direction, the measuring points above the shale shaker show that the air flow velocity at the front of the screen is small, the air flow velocity at the middle is large, and the air flow velocity at the back gradually decreases. When the air flow velocity reaches the tail of the screen, the air flow velocity rises again. This is because the front position above the shale shaker is only affected by the air flow from the air channel on the multichannel centrifugal fan and gradually decreases. The middle part is affected by the air from the lower air duct of the multichannel centrifugal fan, so the speed is relatively high. The tail of the screen face is close to the outlet of the machine, and the cross-sectional area suddenly decreases, resulting in the air flow speed rising. The air flow velocity of each measuring point under the vibrating screen decreases with the increase of the longitudinal distance of the vibrating screen, showing a phenomenon of gradual attenuation. This is because the air flow generated by the lower air duct of the centrifugal fan completely covers the measuring points under the vibrating screen, and the air flow generated by the upper air duct has little impact on these measuring points, so the air flow velocity of the measuring point will gradually decline with the increase of the longitudinal distance of the vibrating screen. The air velocity under the cutting threshing drum is small, and it rises up at the tail, which is due to the air flow blocked by the separation concave.

(2) In addition, the air velocity in the simulation results is $9.3 \%-20.5 \%$ higher than that in the measurement results. This is because temperature, humidity, air viscosity, and other relevant parameters are not considered in the air flow field simulation, resulting in larger results, but the distribution law is still the 


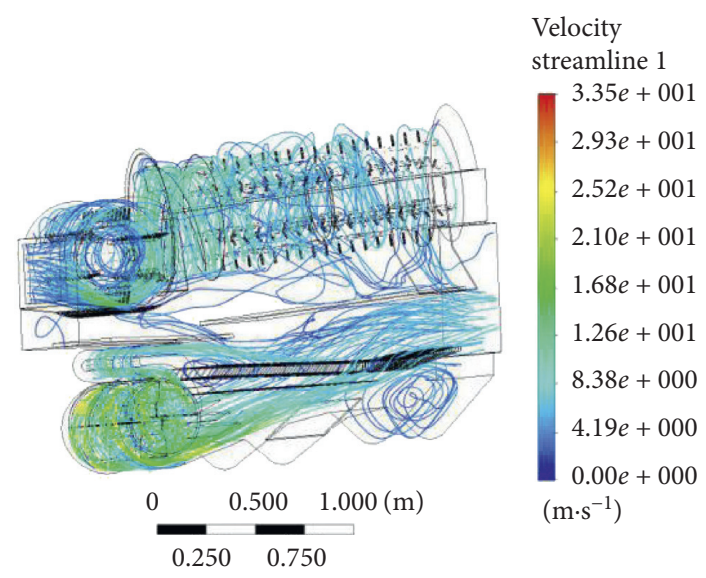

Figure 9: Velocity streamlines diagram.

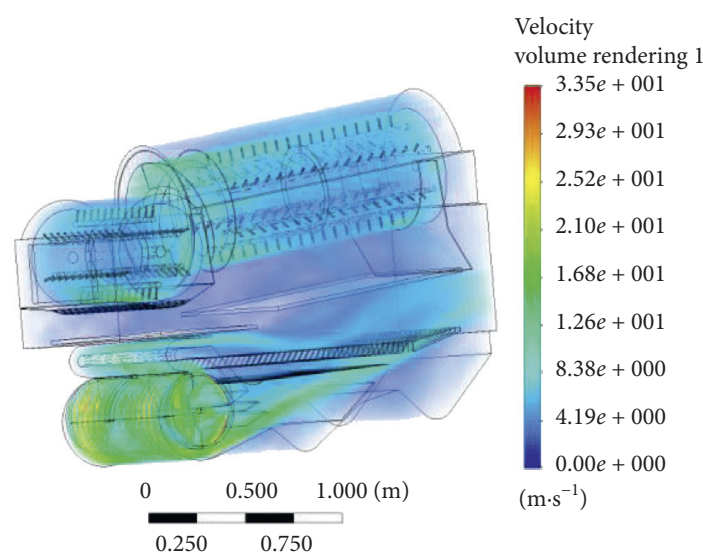

FIGURE 10: Velocity contour diagram.

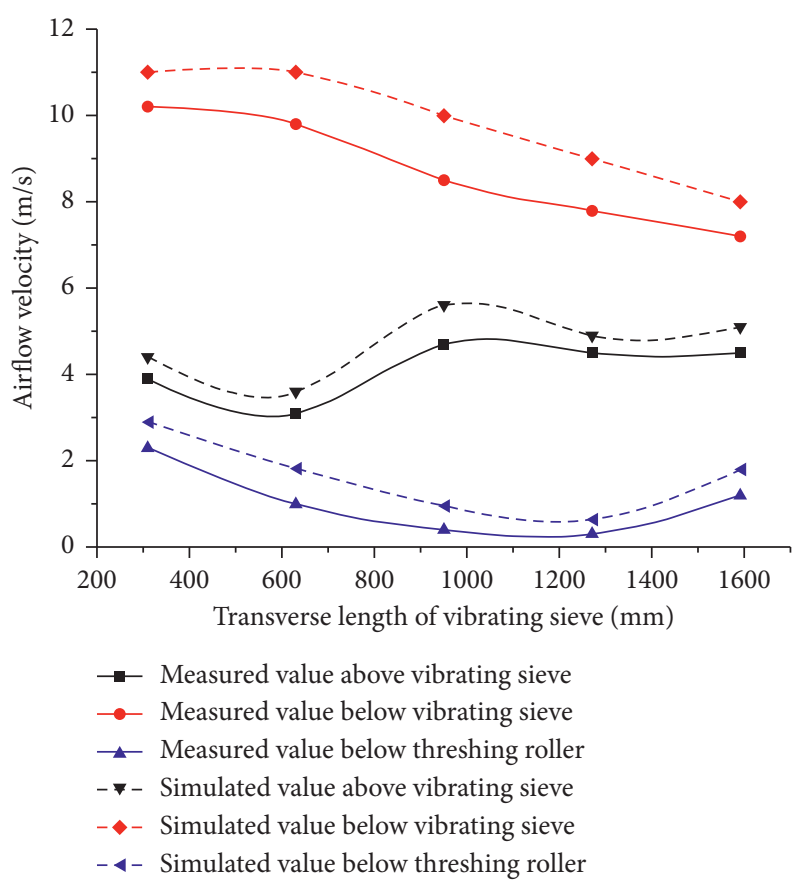

Figure 11: Test and simulation results of air flow velocity measurement. 


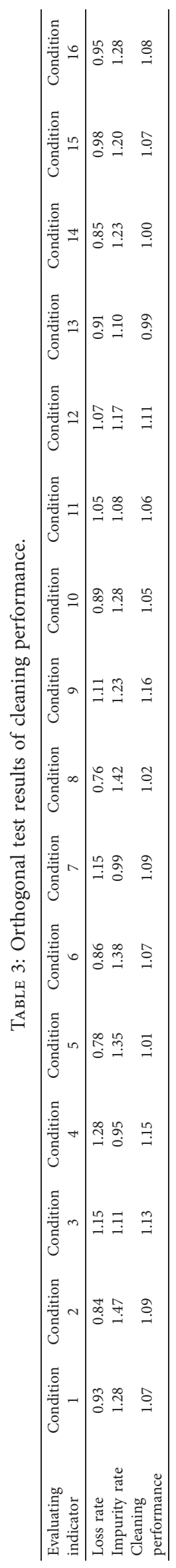




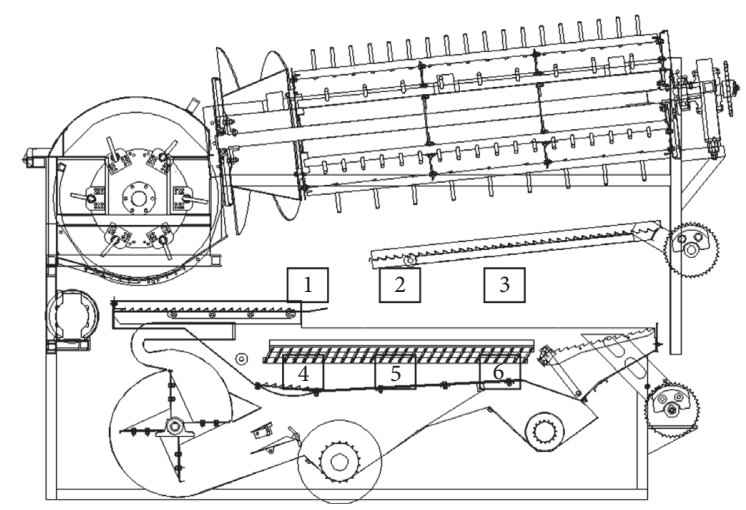

FIGURE 12: Six areas are divided inside the threshing and cleaning device.

same, so it can be used as an analysis factor to establish the cleaning performance prediction model.

3.3. Cleaning Performance Test Results. Cleaning performance is a comprehensive evaluation index obtained by weighted calculation of cleaning loss rate and grain impurity rate. According to the actual harvest demand, the importance of cleaning loss rate is slightly greater than that of grain impurity rate, and the weight coefficients are 0.6 and 0.4 , respectively. The lower the comprehensive evaluation index value is, the better cleaning performance is. The test results are shown in Table 3.

3.4. Prediction Model of Cleaning Performance. From the literature [3], it can be seen that the changes of centrifugal fan speed, angle of air distributor, and opening of chaffer are basic. They will not affect the distribution of the air flow field in the transverse direction of the threshing and cleaning device. Similarly, the impact of the rotation speed of the threshing roller is small. Therefore, in order to establish a more accurate and convenient cleaning performance prediction model, on the basis of the above airflow velocity measurement surface, the threshing and cleaning device is divided into six areas in the longitudinal direction (as shown in Figure 12), and the average airflow velocity of each area can be obtained by using CFX postprocessing software. By analyzing the change of average air velocity in six areas under different working conditions, the mathematical relationship between cleaning performance and its existence is explored.

The average air velocity in 9 areas is taken as independent variable $X$, and the cleaning performance evaluation index is taken as dependent variable $Y$; after analyzing the data, it is found that the average air velocity in area 3 and area 5 is proportional to the cleaning performance evaluation index, so the three areas are analyzed in detail. The polynomial fitting curve between the average air flow velocity of area 3 and area 5 and the cleaning performance evaluation index is made by using Origin software, as shown in Figures 13 and 14.

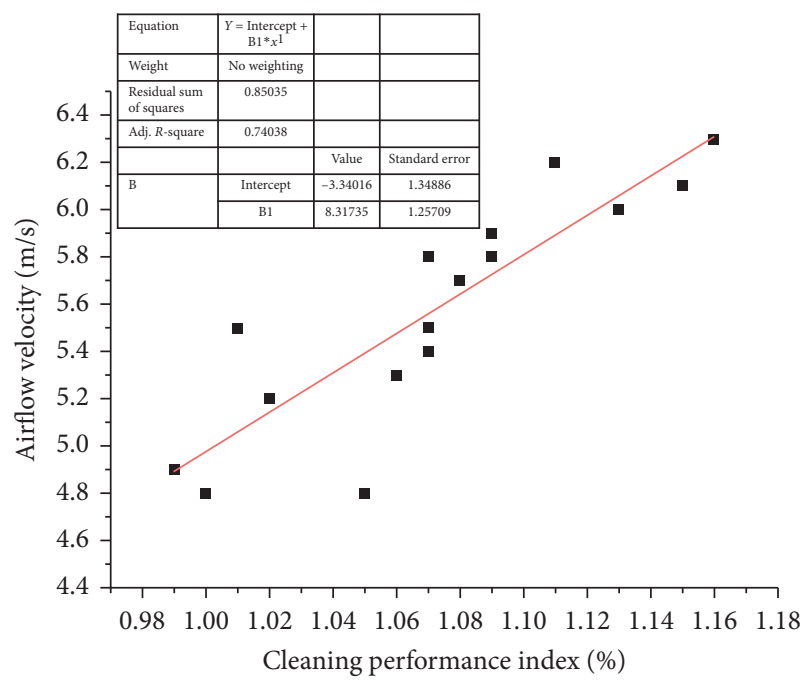

FIGURE 13: Fitting curve of relationship between air flow velocity and cleaning performance in zone 3 .

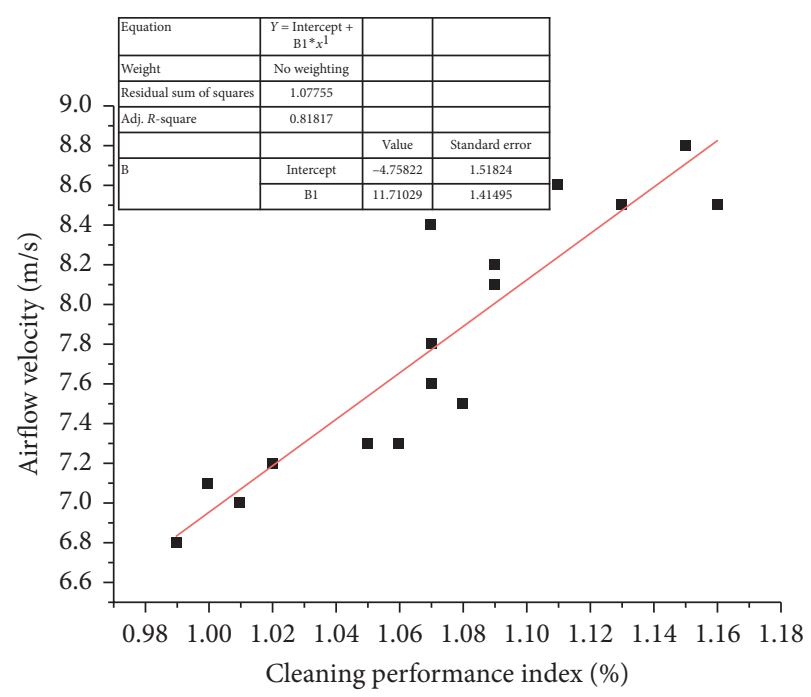

FIGURE 14: Fitting curve of relationship between air flow velocity and cleaning performance in zone 5 .

As can be seen from Figure 14, the Adj. $R^{2}$ value of the fitting curve of the relationship between the average air velocity and cleaning performance in zone 3 is 0.74 , and the Adj. $R^{2}$ value of the fitting curve of the relationship between the average air velocity and cleaning performance in zone 5 is 0.82 . To some extent, the average air velocity and cleaning performance evaluation indexes in the two regions are linearly related. The mathematical function of the relation curve is $Y=11.71 X-4.76$, the relative error of prediction is within $9.4 \%$. This area is located in the middle of the vibrosieve in the threshing and cleaning device, which shows that the air flow velocity in the middle of the vibrosieve is approximately proportional to the cleaning performance within the adjustable range of the working parameters of the designed threshing and cleaning system. 


\section{Conclusion}

The threshing device and cleaning device are taken as a whole to study the air flow field. Taking the rotating speed of centrifugal fan, the angle of air separation plate, the opening of chaffer, and the rotating speed of threshing cylinder of tangential (longitudinal) flow as the research factors, the CFD software is used to simulate and analyze the air flow field in the internal space of the threshing and cleaning system under different working conditions, and the simulation analysis is $9.3 \%-20.5 \%$ larger than the experimental results of air flow field measurement. However, the distribution law of the two is the same, which can be used as the analysis factor to establish the cleaning performance prediction model.

The mathematical relationship between the distribution of air flow field in the threshing and cleaning device and the cleaning performance evaluation index is established. It is found that within the adjustable range of working parameters, the average air flow velocity in the middle of the shale shaker is linearly related to the cleaning performance evaluation index, and the predicted relative error of the relationship function is within $9.4 \%$. This method provides reference for the optimization of the subsequent threshing and cleaning device. Test can also save design time and cost and reduce the influence of seasonality of field test on test cycle.

\section{Data Availability}

The data used to support the findings of this study are available from the corresponding author upon request.

\section{Conflicts of Interest}

The authors declare that they have no conflicts of interest.

\section{Acknowledgments}

This research work was supported by the Research on Quality Inspection Technology of Agricultural Machinery Manufacturing Process of National Key R\&D Plan (2017YFD0700203), National Key R\&D Plan Intelligent Rice Wheat Combine Harvest Technology and Equipment R\&D (2016YFD0702003), and a project funded by the Priority Academic Program Development of Jiangsu Higher Education Institutions (no. PAPD-2018-87).

\section{References}

[1] B. F. Li, Agricultural Mechanics, China Agricultural Press, Beijing, China, 2003.

[2] L. Z. Xu, Y. Xu, and Y. M. Li, "Research progress on cleaning technology and device of grain combine harvester," Transactions of the Chinese Society for Agricultural Machinery, vol. 50, no. 10, pp. 1-16, 2019.

[3] Y. Li, L. Xu, Y. Zhou, B. Li, Z. Liang, and Y. Li, "Effects of throughput and operating parameters on cleaning performance in air-and-screen cleaning unit," Computers and Electronics in Agriculture, vol. 152, no. 5, pp. 141-148, 2018.
[4] P. Ponpesh and D. Giles, "Turbulent gas-particle flow modeling for the mitigation of dust emission from nut harvesters," in Proceedings of the American Society of Agricultural and Biological Engineers Reno, NV, USA, June 2009.

[5] T. B. Anderson and R. Jackson, "A fluid mechanical description of fluidized beds," Industrial \& Engineering Chemistry Fundamentals, vol. 6, no. 4, pp. 527-539, 1967.

[6] H. D. Kutzbach and G. R. Quick, "CGIR handbook of agricultural engineering," in Plant Production Engineering, vol. 3, pp. 27-39, , no. 1, American Society of Agricultural Engineers, St. Joseph, MI, USA, 1999.

[7] G. Craessaerts, J. De Baerdemaeker, B. Missotten, and W. Saeys, "Fuzzy control of the cleaning process on a combine harvester," Biosystems Engineering, vol. 106, no. 2, pp. 103106, 2010.

[8] L. Z. Xu, Y. M. Li, Z. Tang, L. G. Wang, and Z. Y. Jiao, “4LQZ6 tangential-longitudinal axial combine harvester," Transactions of the CSAM, vol. 44, no. 8, pp. 94-98, 2013.

[9] M. G. Gebrehiwot, J. De Baerdemaeker, and M. Baelmans, "Effect of a cross-flow opening on the performance of a centrifugal fan in a combine harvester: computational and experimental study," Biosystems Engineering, vol. 105, no. 2, pp. 247-256, 2010.

[10] Q. Guan, "New products of case's domestic axial-flow drum harvester are on the market," Agricultural Machinery Market, vol. 34, no. 11, pp. 55-56, 2014.

[11] T. Tang, Y. M. Li, and H. C. Li, "Indoor eddy current test of air screen cleaning device of combine harvester," Journal of Agricultural Machinery, vol. 41, no. 12, pp. 62-66, 2010.

[12] Y. Ueka, M. Matsui, E. Inoue, K. Mori, T. Okayasu, and M. Mitsuoka, "Turbulent flow characteristics of the cleaning wind in combine harvester," Engineering in Agriculture, Environment and Food, vol. 5, no. 3, pp. 102-106, 2012.

[13] K. L. Kenney, C. T. Wright, and K. M. Bryden, "Virtual engineering approach to developing selective harvest technologies," Advances in Materials Science and Engineering, vol. 2005, Article ID 056046, 16 pages, 2005.

[14] Y. H. Li, Simulation Research on Air Flow Cleaning Device of Micro Combine Harvester, Nanjing Agricultural University, Nanjing, China, 2008.

[15] B. Yu, Q. Wang, and S. Wang, "Simulation of air flow field of air screen cleaning chamber based on fluent," Jiangsu Agricultural Science, vol. 44, no. 7, pp. 358-361, 2016.

[16] L. J. Yu, Design and Experimental Study of Double Outlet and Multi Air Duct Cleaning Device, Jiangsu University, Zhenjiang, China, 2015.

[17] P. P. Ma, Structure Optimization and Test of Cleaning Device of Single Longitudinal Axial Flow Combine Harvester, Jiangsu University, Zhenjiang, China, 2016.

[18] L. Xu, A. Hansen, Y. Li, Z. Liang, and L. Yu, "Numerical and experimental analysis of airflow in a multi-duct cleaning system for a rice combine harvester," Transactions of the ASABE, vol. 59, no. 5, pp. 1101-1110, 2016.

[19] Z. Liang, Y. Li, J. De Baerdemaeker, L. Xu, and W. Saeys, "Development and testing of a multi-duct cleaning device for tangential-longitudinal flow rice combine harvesters," Biosystems Engineering, vol. 182, no. 7, pp. 95-106, 2019.

[20] L. Zhou and Z. Wang, "Numerical simulation of cavitation around a hydrofoil and evaluation of RNG $\kappa-\varepsilon$ model," Journal of Fluids Engineering, vol. 130, no. 1, pp. 302-308, 2008.

[21] Y. Itazu and Y. Nagano, "RNG modeling of turbulent heat flux and its application to wall shear flows," JSME International Journal Series B, vol. 41, no. 3, pp. 657-665, 1998. 
[22] A. E. Sakya, Y. Nakamura, and M. Yasuhara, "Evaluation of an RNG-based algebraic turbulence model," Computers \& Fluids, vol. 22, no. 2-3, pp. 207-214, 1993.

[23] Y. Yuan, X. Ma, H. Jin, and H. Yin, "Study on vacuum chamber fluid field of air suction seed-metering device for rice bud-sowing," Transactions of the Chinese Society of Agricultural Machinery, vol. 29, no. 6, pp. 42-45, 2005.

[24] J. E. Chen, L. J. Wang, K. Liu, and S. Z. Feng, "Numerical simulation of gas-particle flow in inertia separation chamber of stripper combine harvester," Journal of Jiangsu University, vol. 27, no. 3, pp. 193-196, 2006.

[25] W. C. Song and S. S. Fu, "Principle and application of ultrasonic wind speed and direction measuring device," Electronic Quality, vol. 2016, no. 6, pp. 49-52, 2016. 